\title{
Implante de filtro em veia cava inferior dupla: relato de caso e revisão da literatura
}

\author{
Filter placement in duplicated inferior vena cava: case report and \\ review of the literature \\ Rafael Demarchi Malgor'1, Marcone Lima Sobreira², Priscila Nunes Boaventura ${ }^{2}$, Regina Moura ${ }^{3}$, \\ Winston Bonetti Yoshida ${ }^{4}$
}

\section{Resumo}

Veia cava inferior dupla é uma variação anatômica rara cuja prevalência é de $0,2-3 \%$. O implante de filtro de veia cava, quando indicado em casos com duplicidade da veia cava inferior, pode ser realizado de diferentes formas: em ambas as veias cavas; em uma delas, embolizando a anastomose entre ambas; em somente uma delas; ou por implante supra-renal. Relatamos um caso de trombose venosa profunda no pós-operatório de implante de prótese de quadril com contra-indicação para tratamento anticoagulante e cuja cavografia evidenciou duplicidade de veia cava inferior. O implante de filtro de veia cava inferior realizado em posição supra-renal mostrou-se opção adequada e segura.

Palavras-chave: Veia cava inferior, filtros de veia cava, anormalidades

\section{Introdução}

Duplicidade da veia cava inferior (VCI) é uma malformação rara, com prevalência entre $0,2-3 \%^{1-3}$. O conhecimento dessa variação anatômica se torna importante em casos de reparos cirúrgicos tanto na VCI como em suas adjacências ${ }^{4,5}$.

O diagnóstico da duplicação da VCI, na grande maioria dos casos, é realizado por achados de exames complementares, uma vez que os sinais ou sintomas são raros durante a vida do paciente. O implante de filtro em VCI pode ser realizado com controle radioscópico, que é o método de escolha. Alguns autores também têm descrito a realização do implante de filtro com controle ultra-sonográfico quando há duplicidade de cava ${ }^{6-8}$.

\begin{abstract}
Double inferior vena cava is a rare anatomic variation with prevalence ranging between $0.2-3.0 \%$. In cases of duplication, inferior vena cava filter placement options include placing it in both vena cava, coil-embolization of the intervenous segment plus placing a filter in the right inferior vena cava, or suprarenal filter placement. We report a case of deep venous thrombosis after unilateral primary total hip replacement, presenting with contraindications for anticoagulant therapy, in which cavography showed inferior vena cava duplication. Inferior vena cava filter placement was performed in the supra-renal portion and was proved to be an adequate and safe procedure.
\end{abstract}

Keywords: Vena cava inferior, vena cava filters, abnormalities

São escassos relatos de implante de filtro de veia cava nessa situação. Relatamos um caso de implante de filtro de cava em posição supra-renal em paciente com duplicidade de VCI.

\section{Descrição do caso}

Trata-se de paciente de 74 anos, sexo feminino, hipertensa, obesa (índice de massa corporal $=34,92$ ), submetida a artroplastia de quadril direito. A profilaxia medicamentosa (heparina não-fracionada a 5000 UI a cada 8 horas por via subcutânea) para tromboembolismo venoso foi prescrita, iniciando-se 12 horas após a cirurgia. No $30^{\circ}$ dia pós-operatório, devido a distúrbio de coagulação ocasionado durante a administração

1. Médico residente, Serviço de Cirurgia Vascular, Departamento de Cirurgia e Ortopedia, Universidade Estadual Paulista (UNESP), Botucatu, SP.

2. Médico contratado, Serviço de Cirurgia Vascular, Departamento de Cirurgia e Ortopedia, UNESP, Botucatu, SP.

3. Doutora. Professora adjunta, Disciplina de Cirurgia Vascular, Departamento de Cirurgia e Ortopedia, UNESP, Botucatu, SP.

4. Doutor. Professor livre-docente, Disciplina de Cirurgia Vascular, Departamento de Cirurgia e Ortopedia, UNESP, Botucatu, SP.

Não foram declarados conflitos de interesse associados à publicação deste relato de caso.

Artigo submetido em 06.01.08, aceito em 11.04.08. 


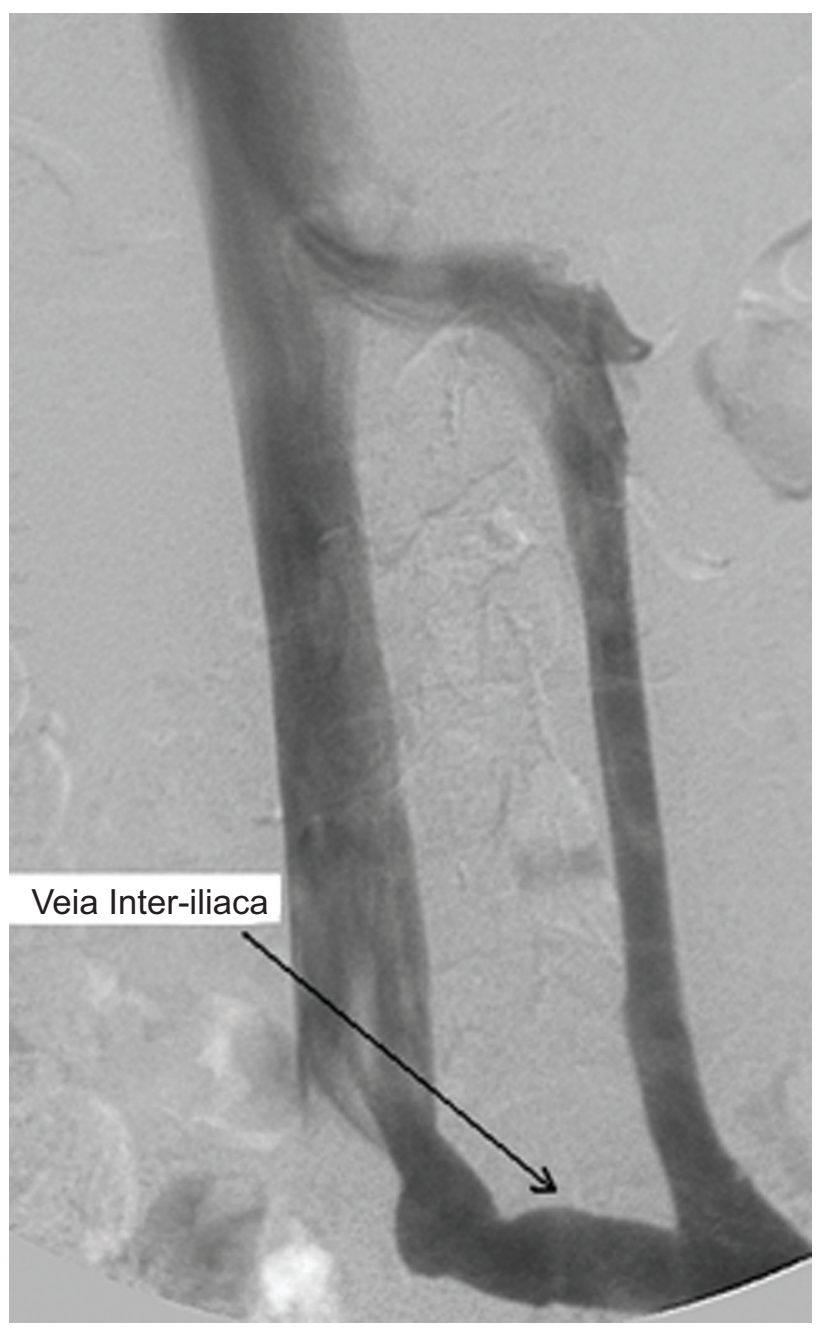

Figura 1 - Cavografia por cateterismo femoral esquerdo evidenciando veia cava dupla (seta indicando veia interilíaca)

domiciliar de heparina, a paciente foi novamente admitida na enfermaria de ortopedia, onde teve o tratamento suspenso. Após sangramento do sítio cirúrgico, instalou-se processo infeccioso local causado por Acinetobacter $\mathrm{sp}$, inicialmente tratado com imipenem /cilastatina. Cinco dias após a suspensão da profilaxia para tromboembolismo venoso, em tratamento por infecção da ferida operatória, acamada, a paciente evoluiu com quadro de dor e edema assimétrico em todo o membro inferior direito; não se observou dispnéia ou sinais sugestivos de tromboembolismo pulmonar.

O mapeamento dúplex venoso de membros inferiores mostrou imagens de trombose venosa profunda das veias femoral, poplítea, tibiais posteriores e fibulares em membro inferior direito, com ausência de trombose venosa profunda em membro inferior esquerdo. Os exames laboratoriais eram normais, assim como raio $\mathrm{X}$ de tórax, eletrocardiograma e tomografia computadorizada de tórax.

Interrompida a profilaxia com heparina devido ao sangramento e estando programada cirurgia de desbridamento da ferida operatória, a paciente foi considerada de alto risco para embolia, e por isso the foi indicado o implante de filtro de VCI.

Após punção de veia femoral comum esquerda e inserção de bainha de $6 \mathrm{Fr}$, observou-se, na venografia, duplicação de VCI desde o início da confluência das veias ilíacas, inclusive com presença de veia interilíaca, até o nível da entrada da veia renal direita (Figura 1).

Tendo em vista o espaço adequado em veia cava supra-renal, optou-se por implante de filtro de veia cava (VenaTech LP -B, Brown, Nashville, EUA) nessa posição, com passagem do aplicador do filtro desde a veia femoral esquerda previamente abordada e através da cava esquerda (Figura 2).

A paciente evoluiu sem intercorrências em pósoperatório imediato ao implante e sem sinais de tromboembolismo pulmonar pós-cirurgia de desbridamento

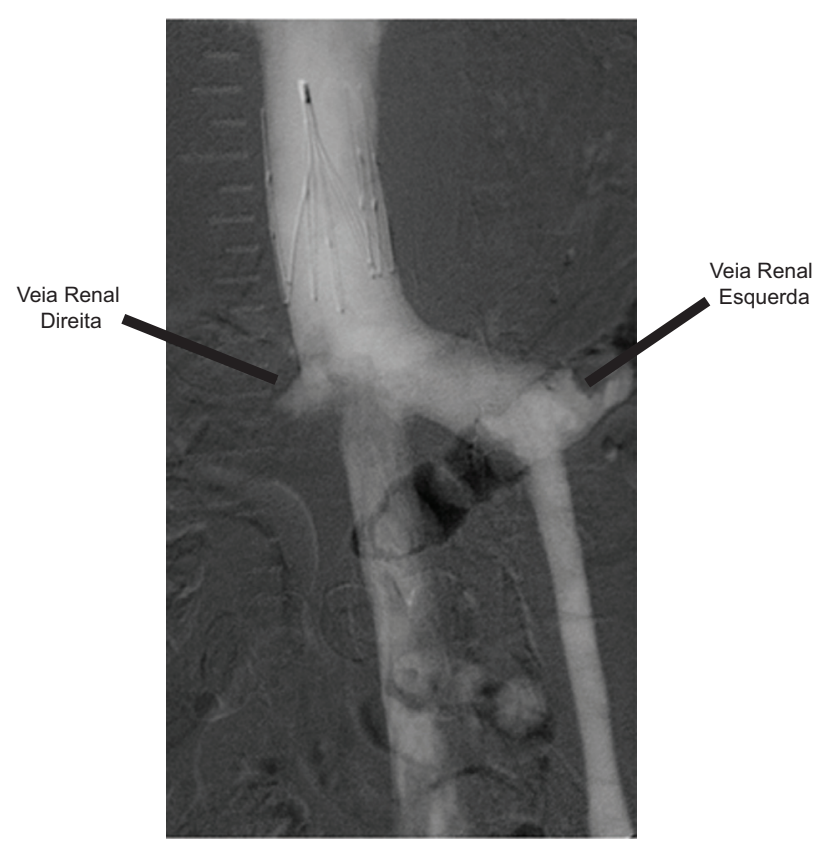

Figura 2 - Implante supra-renal de filtro em veia cava inferior dupla 
em região coxo-femoral direita. Devido à infecção da prótese de quadril, a paciente permaneceu por 60 dias internada e recebeu alta hospitalar sem intercorrências relacionadas ao procedimento de implante de filtro de VCI durante a internação.

\section{Discussão}

A duplicação de veia cava pode se apresentar de várias formas: VCI retroureteral, VCI esquerda, VCI retrocaval, VCI com drenagem pela veia ázigos e interrupção da VCI com continuação pela veia ázigos ${ }^{9}$. A duplicidade ocorre quando há falha de fusão das veias cardinais durante a embriogênese ou não regressão da veia supracardinal esquerda ${ }^{10}$.

A prevalência de veia cava dupla é baixa, e sua associação com trombose venosa e indicação de filtro é ainda mais rara ${ }^{11}$. Somente seis casos de implante de filtro em veia cava dupla foram descritos até o momento. As indicações de implante de filtro de VCI nesses casos foram: 1) contra-indicação de anticoagulação, três casos (sangramento e neurocirurgia recente) ${ }^{11}$; 2) prevenção de tromboembolismo pulmonar maciço em casos de trombose venosa profunda proximal extensa, dois $\operatorname{casos}^{11}$; 3 ) tromboembolismo pulmonar em vigência de anticoagulação, um caso ${ }^{11}$.

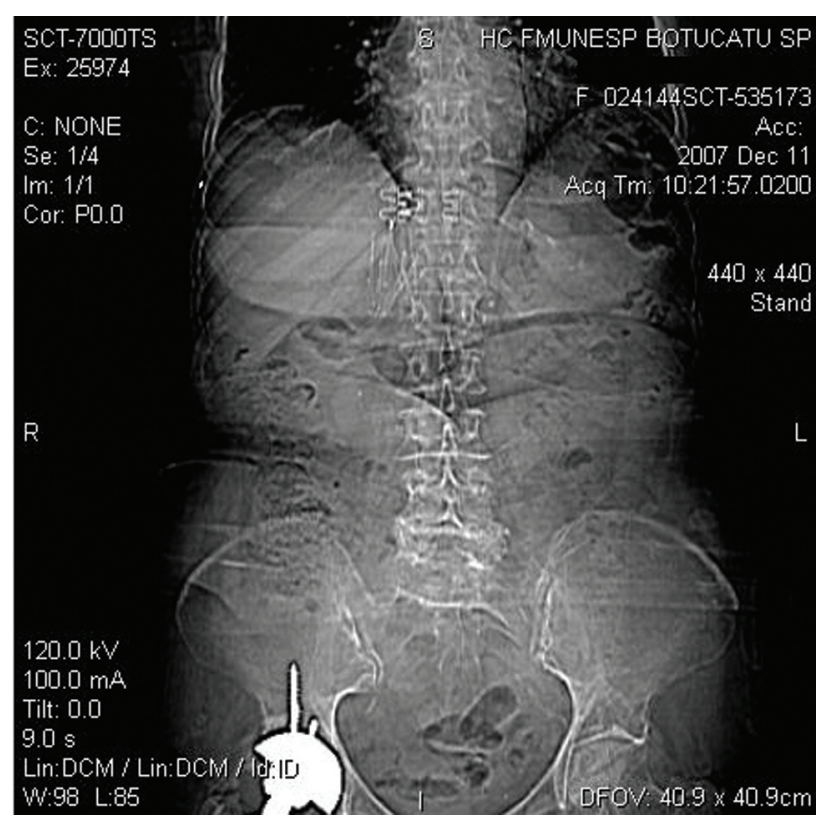

Figura 3 - Raio X de abdome evidenciando filtro de veia cava 6 meses após o procedimento

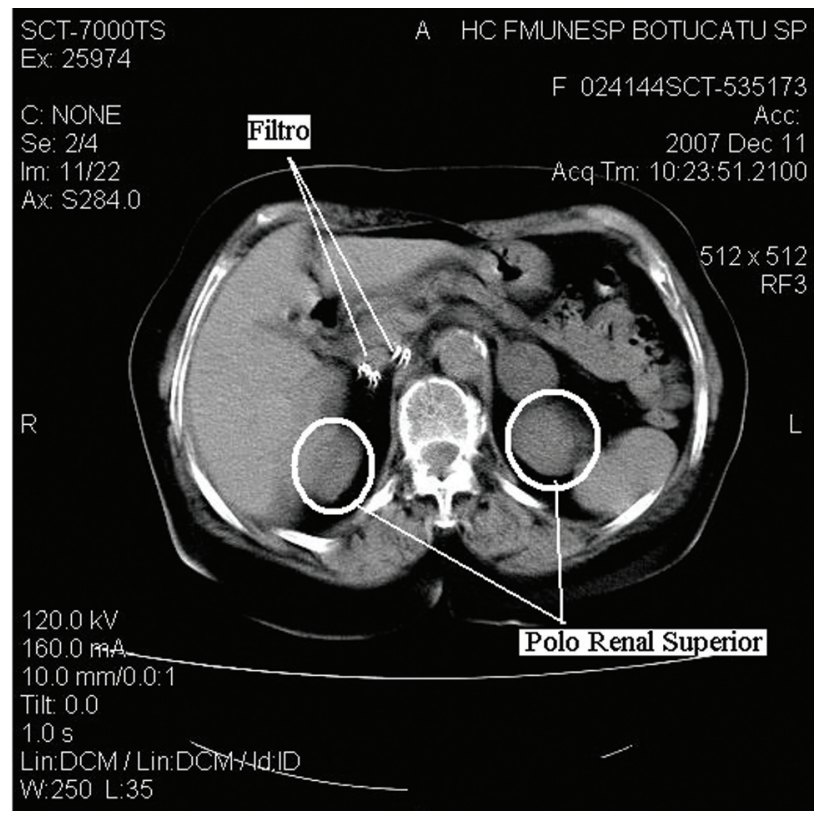

Figura 4 - Tomografia computadorizada evidenciando porção final de filtro em posição supra-renal (veia cava inferior pérvia, seguimento de 6 meses)

Os filtros implantados foram: Kimray Greenfiled (Medi-Tech/Boston Scientific, Watertoen, EUA), Simon Nitinol (C.R. Bard, Murray Hill, EUA), Vena Tech LP (B/Brown, Bethlehem, EUA). Em três casos, os filtros foram implantados nos dois ramos da cava; em um caso, no ramo direito, paralelamente a embolização do ramo esquerdo; e em dois casos, em posição supra-renal.

Dentre as opções de local de implante ${ }^{12-14}$, talvez a posição escolhida neste caso seja a mais interessante porque evita procedimentos adicionais como embolização de uma das veias cavas ou de sua interligação. Além disso, somente um filtro é utilizado, representando uma economia expressiva, já que, em nosso meio, os materiais endovasculares são importados e portanto muito caros.

A liberação do filtro em veia cava supra-renal é segura e já está consagrada em situações especiais. Greenfield et al. têm preconizado o implante de filtro de VCI em posição supra-renal quando há trombos se estendendo acima do nível das veias renais ou dentro delas, em pacientes gestantes ou em mulheres em idade férti ${ }^{14}$. Tem sido também descrito o implante suprarenal em casos de veia cava com pequeno calibre ${ }^{15}$. 
A necessidade de anticoagulação após implante de filtro é discutível. Decousus et al. ${ }^{16}$, em estudo randomizado, mostrou que os pacientes com filtro e anticoagulados tiveram recorrência de tromboembolismo pulmonar maior a longo prazo do que os somente anticoagulados. Sendo assim, é possível que pacientes com filtro e não anticoagulados possam ter taxas de recorrência ainda maiores. Por outro lado, consenso recente não recomenda o uso de anticoagulantes a longo prazo nesses pacientes. A paciente do presente relato não foi anticoagulada após implante do filtro ${ }^{17}$.

Levou-se em consideração, na escolha do tipo de filtro, o perfil e a facilidade de aplicação. Como o introdutor atravessou facilmente a veia cava esquerda (menos calibrosa), o implante de filtro com baixo perfil foi facilitador do procedimento. Entretanto, outros modelos ou mesmo filtros temporários poderiam ser igualmente efetivos, inclusive com a opção de acesso pela veia jugular.

A paciente em questão segue assintomática após 6 meses, sem intercorrências inerentes ao procedimento (Figuras 3 e 4). Tal evolução sugere que o implante supra-renal de filtro de veia cava pode ser uma opção segura e efetiva quando existe esse tipo de duplicação de veia cava.

\section{Referências}

1. Bartle EJ, Pearce WH, Sun JH, Rutherford RB. Infrarenal venous anomalies and aortic surgery: avoiding vascular injury. J Vasc Surg. 1987;6:590-3.

2. Chuang VP, Mena CE, Hoskins PA. Congenital anomalies of the inferior vena cava. Review of embriogenesis and presentation of a simplified classification. Br J Radiol. 1974;47:20613 .

3. Bass JE, Redwine MD, Kramer LA, Huynh PT, Harris JH Jr. Spectrum of congenital anomalies of the inferior vena cava: cross-sectional imaging findings. Radiographics. 2000;20:63952.

4. Sam AD 2nd, Frusha JD, McNeil JW, Olinde AJ. Repair of a blunt traumatic inferior vena cava laceration with commercially available endografts. J Vasc Surg. 2006;43:841-3.

5. Oderich GS, Panneton JM, Hofer J, et al. Iatrogenic operative injuries of abdominal and pelvic veins: a potentially lethal complication. J Vasc Surg. 2004;39:931-6.
6. Uppal B, Flinn W, Benjamin ME. The bedside insertion of inferior vena cava filters using ultrasound guidance. Perspect Vasc Surg Endovasc Ther. 2007;19:78-84.

7. Passman MA, Dattilo JB, Guzman RJ, Naslund TC. Bedside placement of inferior vena cava filters by using transabdominal duplex ultrasonography and intravascular ultrasound imaging. J Vasc Surg. 2005;42:1027-32.

8. Neser RA, Capasso Filho M, Homa CMO. Implante de filtro de veia cava inferior guiado por ultra-som: relato de dois casos. J Vasc Bras. 2006;5:71-3.

9. Xue HG, Yang CY, Asakawa M, Tanuma K, Ozawa H. Duplication of the inferior vena cava associated with other variations. Anat Sci Int [revista eletrônica]. 2006 Jul [citado 2006 jul 03] [aproximadamente 5 páginas]. Disponível em: http://www.anatomy.or.jp/e-top.html.

10. Anne N, Pallapothu R, Holmes R, Johnson MD. Inferior vena cava duplication and deep venous thrombosis: case report and review of Literature. Ann Vasc Surg. 2005;19:740-3.

11. Sartori MT, Zampieri P, Andres AL, Prandoni P, Motta R, Miotto D. Double vena cava filter insertion in congenital duplicated inferior vena cava: a case report and literature review. Haematologica. 2006;91(6 Suppl):ECR30.

12. Matchett WJ, Jones MP, McFarland DR, Ferris EJ. Suprarenal vena caval filter placement: follow-up of four filter types in 22 patients. J Vasc Interv Radiol. 1998;9:588-93.

13. Putnam SG 3rd, Ball D, Cohen GS. Placement of bilateral Simon Nitinol filters for an inferior vena caval duplication through a single groin access. J Vasc Interv Radiol. 1999;10:431-3.

14. Greenfield LJ, Cho KJ, Proctor MC, Sobel M, Shah S, Wingo J. Late results of suprarenal Greenfield vena cava filter placement. Arch Surg. 1992;127:969-73.

15. McIntosh S, Brautigam R, Gross R. Duplicate inferior vena cava. J Trauma. 2006;61:235.

16. Decousus H, Leizorovicz A, Parent F, et al. A clinical trial of vena caval filters in the prevention of pulmonary embolism in patients with proximal deep-vein thrombosis. Prévention du Risque d'Embolie Pulmonaire par Interruption Cave Study Group. N Engl J Med. 1998;338:409-15.

17. British Committee for Standards in Haematology Writing Group, Baglin TP, Brush J, Streiff M. Guidelines on use of vena cava filters. Br J Haematol. 2006;134:590-5.

Correspondência:

Rafael Demarchi Malgor

Departamento de Cirurgia e Ortopedia, UNESP

Rubião Júnior $\mathrm{s} / \mathrm{n}^{\circ}$

CEP 18600-400 - Botucatu, SP

E-mail: rafaeldemarchi@yahoo.com.br 